\title{
A STOCHASTIC FORMATION OF RADIATIVE TRANSFER IN CLOUDS
}

\begin{abstract}
The research carried out under this award dealt with issues involving (1) deterministic radiative transfer, (2) remote sensing, (3) Stochastic radiative transfer, and (4) parameterization of cloud optical properties. A number of different forms of radiative transfer models in one, two, and three dimensions were developed in an attempt to build an understanding of the radiative transfer in clouds with realistic spatial structure and to determine the key geometrical parameter that influence this transfer. The research conducted also seeks to assess the relative importance of these geometrical effects in contrast to microphysical effects of clouds. The main conclusion of the work is that geometry has a profound influence on all aspects of radiative transfer and the interpretation of this transfer. We demonstrate how this geometry can influence estimate of particle effective radius to the $30-50 \%$ level and also how geometry can significantly bias the remote sensing of cloud optical depth.

We haven't yet identified the main way of parameterizing these geometric effects but we have developed a stochastic form of radiative transfer and have explored the possibility of incorporating gross statistics of clouds in simple radiative transfer models. We have also developed a very useful parameterization of cloud optical properties which will also ultimately be incorporated into this simple model. We propose that this model will serve as a framework for a new cloud-radiation parameterization for global models.
\end{abstract}

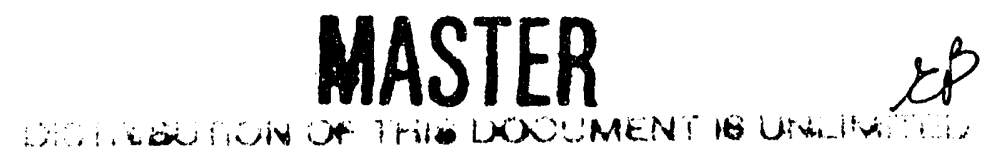




\section{Introduction}

The research conducted as part of this project breaks down into four broad areas:

- Deterministic Radiative Transfer

- Remote Sensing

- Stochastic Radiative Transfer

- Parameterization of cloud optical properties

The approach pursued in this research is to employ different forms of radiative transfer models in one, two, and three dimensions in an attempt to build an understanding of the radiative transfer in clouds with realistic spatial structure and to determine the key geometrical parameters that influence this transfer. A key focus is the understanding of the relative importance of these geometrical effects in contrast to microphysical effects of clouds. The main conclusion is that geometry has a profound influence on all aspects of radiative transfer and the interpretation of this transfer. We haven't yet identified the main way of parameterizing these geometric effects but we have developed a very useful parameterization of cloud optical properties which will ultimately be incorporated into a parameterization.

\section{Deterministic Radiative Transfer}

Our research on radiative transfer has produced three different types of solutions to the radiative transfer equation. The first is an efficient Monte Carlo model (O'Brien 1992) that has been used as a benchmark for testing the other two nu- 
merical models. The two different numerical solutions are in excellent agreement with Monte-Carlo results. These models are discussed in detail by Gabriel et al. (1993) for the Fourier-Riccati method and Evans (1993) for the Spherical Harmonic Spatial Grid method.

The Fourier-Riccati model was used to study the effects of geometry on solar heating rate. The essential conclusions of this work are given in Fig. 1 showing the horizontal disposition of the heating at different depths in the cloud compared with the plane parallel heating. The heating is highly distributed and significantly exceeds the plane parallel values in the most dense regions of the cloud. The two panels differ in the microphysics assumed in the simulation. Clearly both microphysics and geometry affect the heating in important ways.

\section{Remote Sensing}

We also examine the the effects of geometry on spectral reflectance and interpret these effects in terms of retrieved optical properties of clouds using procedures published in the literature. An example of this work is presented in Fig. 2 which shows the bispectral plot of radiances derived from a simulation of radiative trausfer through a 2D variable cloud. The particle size distribution was fixed in this cloud and only number concentration was varied (thus the effective radius was also fixed at all locations). The scatter of points of Fig. 2 is a direct result of the geometrical influences on spectral reflectances. This scatter is erroneously interpreted as changes in both optical depth and effective radius, the former varying by as much as 20 percent and the latter by as much as 30-50 percent. 


\section{Stochastic Radiative Transfer}

It is clear from these and many other studies that geometrical factors profoundly influence the transfer of radiation through clouds. It is unlikely that all of the relevant scales of spatial variability will be resolved in climate models, or even cloud models. Furthermore, it is the mean rediative quantities and not the details of the radiation field that are important for the climate system. The question remains as to what is the relevant structure information and how this information should be incorporated into radiative transfer. Stochastic transfer methods attempt to address these questions by investigating the radiative effects of ensembles of cloud structures described by probability distributions. The previous work on stochastic radiative transfer has been limited in its applicability to the atmosphere by assumptions such as small amounts of variability, no internal structure in clouds, or insppropriate spatial distribution functions.

We have developed a new method of stochastic radiative transfer that applies to any sort of spatial variability in clouds (Evans 1993). The new method is based on the backward Monte Carlo solution of radiative transfer, using O'Brien's (1992) method. The radiance or flux exiting a surface is expressed as an order of scattering series. Each scattering is associated with an integral over transmission along a path and an angular integral over direction to the next path. The fundamental idea behind this new method of stochastic radiative transfer modeling is to do backward Monte Carlo integration of the order of scattering integral with additional integrations over probability distributions of path lengths. The spatial variability 
information is expressed in path probability density functions. With the assumption of homogeneous and isotropic statistics a single path pdf has the form $\mathrm{f}(\mathrm{s}-\mathrm{T})$, which is the conditional density of distance (s) given transmission (T). Since successive paths are not independent, higher order information such as the two path pdf $f\left(s_{2} \mid T_{2}, s_{1}, T_{1}\right)$ is needed. There is a hierarchy of approximations based on the order and form of the path pdf's.

The accuracy of these approximations was tested by computing deterministic Monte Carlo radiative transfer in many realizations of 3D isotropic lognormalmultifractal extinction fields. In simulations of thermal radiative transfer in cirrus clouds and solar radiative transfer in boundary layer clouds, each with a range of variability, it was found that the stochastic method with two path pdf's gave accurate mean flux results, while use of the single path pdf's usually did not. Besides providing a practical and general method of stochastic radiative transfer, this work also indicates what cloud structure information is important for radiative transfer. Path pdf's can be obtained from in situ measurements using aircraft as well as from cloud radars and lidars. Such observations combined with further modeling should lead to radiation parameterizations for climate models that incorporate the effect of heterogeneities in clouds.

\section{Parameterization of optical properties}

We have developed a general parameterazation of the optical properties of cloud particles - both of spherical and non-spherical particles. These parameterizations are being geared toward use in global climate models. The scheme under development 
includes:

- fast solvers of the radiative transfer equation based on the two stream version of the model. These solvers were discussed in an earlier study (Flatau et al., 1990) and are now being implemented together with new scattering parameterizations.

- explicit model of cloud optical properties capable of differentiating water and various classes of ice. The parameterization developed is based on van de Hulst's anomalous diffraction theory and the Bohren and Nevit's approximation. The method approximates efficiencies in terms of known functions and in terms of the parameter $v=2 \pi r n^{\prime} / \lambda$ where $n^{\prime}$ is the complex part of the refractive index. In this study, it is discovered how $v$ acts as a similarity parameter reducing the scattering properties of particles of different size $r$ and composition $\left(n^{\prime}\right)$ to a common problem. These results are extremely powerful as they allow us to model the optical properties of clouds in terms of the composition of particles and the size and shape. This work is currently being completed and papers describing it are in the process of being written.

6. List of publications both in progress and completed that acknowledge this award

Duda, D., G. L. Stephens, K. F. Evans, and W. R. Cotton, 1993: The effect of microphysics on the albedo and reflectivity of two dimensional cloud fields. In preparation: J. Atmos. Sci.

Duda, D. and G. L. Stephens, 1993: Radiative heating in two dimensional cloud fields. In preparation: J. Atmos. Sci. 
Evans, K. F., 1993. Two-dimensional Radiative Transfer in Cloudy Atmospheres: The Spherical Harmonic Spatial Grid Method. In press: J. Atmos. Sci.

Evans, K. F., 1993: A General Solution to the Stochastic Radiative Transfer Problem. In preparation.

Evans, K. F. and G. L. Stephens, 1993: A Theoretical Foundation for Microwave Remote Sensing of Cirrus Clouds from Space. In preparation for: J. Atmos. Sci.

Gabriel, P. , S-C Tray, and G. L. Stephens, 1993: A Fourier Riccati Approach to Radiative Transfer Part I: Foundations. In press: J. Atmos. Sci.

O'Brien, D. M., 1992: Accelerated quasi-Monte Carlo integration of the radiative transfer equation. J. Quant. Spectrosc. Radial. Transfer, 48, 41-59.

Tray, S-C, Gabriel, P., and G. L. Stephens, 1993: A Fourier Riccati Approach to Radiative Transfer Part 2: Applications. In preparation: J. Atmos. Sci.

Stephens, G. L., 1993: The remote sensing of the lower atmosphere: An Introducion. Oxford Uni. Press. 540pp. To appear Summer 1993.

7. Budget Comment

There will be no residual funds.

Reprints + Pregrients remove 


\section{Remote Sensing \\ of the Lower Atmosphere}

An Introduction

Graeme L. Stephens

Colorado State University

New York Oxford

OXFORD UNIVERSITY PRESS

1993 


\section{Preface}

With its growth, the atmospheric sciences have become increasingly complicated forcing the working scientist to specialize in one discipline or another. This especially applies to the subject of remote sensing. Yet remote sensing crosses over the imaginary disciplines of the science and is found in one form or other in most studies of the atmosphere. Data derived from remote sensing systems are not only incorporated into both the initialization and validation of forecast models, for example, but are now used extensively in studies that seek to gain fundamental new knowledge about the workings of the atmosphere and the Earth's climate system. This book has grown out of the belief that it is now almost essential, not only for practicing meteorologists but also for the scientist who is to devote his career to the study of the atmosphere, to familiarize him- or her-self with general topics on remote sensing. While the book is intended for both graduate students aud atmospheric scientists, it may also be useful to experts on the subject of remote sensing who seek to place their speciality in a broader verspective.

Interest in the remote sensing a the atmosphere has blossomed. The number of articles scattered in the literature representing various disciplines must run to thousands; there are special journals devoted to the subject and numerous books that deal with various topics of remote sensing are available to the interested scientist. The last time I counted, I found 27 of these books on my shelf alone. This is another book about remote sensing. Given the apparent abundance of books on the topic, an obvious question to ask is how does this book differ from others? I believe the difference is one of emphasis. This book focuses on understanding the basic interactions between radiation and the atmosphere rather than on cataloging numerous mathematical recipes. It is more conceptual and about a way of thinking of these interactions as much as it is a dialogue about remote sensing. Most remote sensing books are specialized in one way or another. I have books that address only the remote sensing associated with a specific technology (such as radar), or a specific platform, such as "satellite remote sensing", or which focus on a specific class of properties such as surface properties. It was never my intention to present the newest metiods of remote sensing in this 
book, nor was it my intention to emphasize the most recent developments of sensor systems, imaging systems or platforms as this would surely date the book before ever reaching the reader. My aim is to describe the basic interactions between electromagnetic radiation and matter and to highlight these interactions using examples drawn from remote sensing. This book was written with the belief that an understanding of these interactions provides a solid foundation for understanding not only other topics of remote sensing but also the processes that govern the distribution of radiation in the Earth's atmosphere.

In writing this book I grew to appreciate the commeuts of professor Peter V. Hobbs who, in reference to his own writings (Hobbs, 1974), remarked on how one never finishes a book of this type but merely abandons it. I finally abandoned this book in 1992. I realized from the beginning the futility in writing a comprehensive text on all the significant aspects of remote sensing. Omission of certain topics was obviously necessary as was the omission of various references to literature. I hope my colleagues will forgive me if my sense of priorities in selection does not match their own. I am guilty of committing a grave disservice to inversion theory, I have largely ignored the remote sensing of ocean properties, I have overlooked the remote sensing of energy fluxes, including the top of the atmosphere radiative budget, I have omitted the growing technique of Raman sensing and refrained from highly technical discussions about the operation and performance of selected instrumentation.

This book, grown from two graduate courses taught in the Department of Atmospheric Sciences at Colorado State University, is intended both as an introductory text on atmospheric remote sensing and as a supplement to topics on atmospheric radiation. The topics discussed in the book are divided into two main sections. After the introductory chapter, the basic properties of radiation and how it interacts with matter are presented in chapters 2 to 5 . Chapters 6 and 7 build on these topics and, after a brief introduction to the subject of radiative transfer, focus on topics of passive remote sensing of various atmospheric parameters. The final chapter discusses the topic of active sensing and includes the subjects of radar and lidar sensing. The bibliographical notes at the end of each chapter list some historical papers and other important papers, relevant books and review articles as well as special papers that offer specific details about the topic in question. These references are a matter of personal judgement and, while the list is not extensive, should 
provide the interested reader with a starting point for his or her own literature search. The problems at the end of each chapter are more didactic in nature than perhaps typical of practical problems encountered in remote sensing. Five more substantial projects, involving analyses of real data, are included in Appendix 2. The aim of these is to expose the interested student to some af the more practical problems one encounters in remote sensing. Details about how these data may be obtained are also provided in the Appendix.

Numerous colleagues contributed to this text invarious ways. A number read selected chapters of the manuscript and suggested improvements in the presentation. One of my colleagues and a friend painstakingly read the entire manuscript in an effort to free it from errors and I an in your debt. My graduate students and students from different classes were also inflicted with the task of working through most of the problems and helped massage many of them into a meaningful form. A few of my graduate students also substantially contributed to the projects listed in the Appendix. I will not mention specific individuals since you know who you are. This book took several years to organize and write. The time it took, and the time I did not have, was taken from my family. To my family I apologize and thank you for your understanding. I know that I can never give this time back. 


\section{Contents}

\section{Introductory Surveys ...................}

1.1 General Classifications..$\ldots \ldots \ldots \ldots \ldots \ldots \ldots \ldots \ldots \ldots \ldots$

1.2 The Nature of Inverse Problems $\ldots . \ldots \ldots \ldots \ldots \ldots \ldots \ldots . \ldots . \ldots . \ldots$

Excursus: The ill-posed nature of

the inversion problem $\ldots \ldots \ldots \ldots \ldots \ldots \ldots \ldots \ldots \ldots . \ldots \ldots$

1.3 The chemical composition of the atmosphere ...........9

1.4 Vertical distribution of pressure and density $\ldots \ldots \ldots \ldots \ldots 12$

1.5 The thermal structure of the atmosphere $\ldots \ldots \ldots \ldots \ldots \ldots 14$

Excursus: Microwave measurements of

atmospheric temperature ..................... 14

1.6 The particulate composition of the atmosphere $\ldots \ldots \ldots \ldots 16$

(a) Atmospheric Aerosol .......................... 18

(b) Cloud microphysics .......................... 19

1.7 Satellite Platforms $\ldots \ldots \ldots \ldots \ldots \ldots \ldots \ldots \ldots \ldots \ldots \ldots . \ldots \ldots$

Excursus: Mechanics of a satellite orbit.............24

(a) Satellite Orbits............................25

(b) Selected Historical Highlights ................... 29

1.8 Notes and Comments ........................... 31

\section{The Nature of Electromagnetic}

Radiation .....................................33

Excursus: The Electric Dipole ................. 33

2.1 The Electromagnetic Spectrum ................... 34

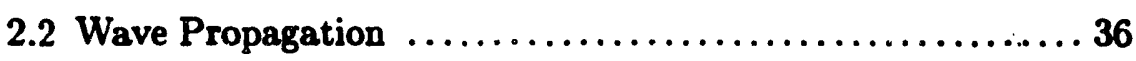

(a) Mathematical Description ........................ 36

Excursus: The Intensity and Irradiance of Electromagnetic Radiation .................... 39 
(b) Waves in Three Dimensions .................. 41

(c) Doppler Effect ........................... 42

2.3 Polarization $\ldots \ldots \ldots \ldots \ldots \ldots \ldots \ldots \ldots \ldots \ldots \ldots \ldots \ldots \ldots \ldots, \ldots \ldots$

(a) Mathematical Description ...................46 46

(b) Examples of Polarized Radiation ...............50

2.4 Stokes' Parameters ..............................5 52

Excursus: A Circular Basis for the

Description of Polarization ....................54

(a) Measurement of $I, Q, U$ and $V \ldots \ldots \ldots \ldots \ldots \ldots \ldots \ldots, \ldots \ldots$

Excursus: Example Calculation of $I, Q, U$ and $V$.....60

Excursus: Mueller Matrices .....................62

2.5 Creation of an Electromagnetic Wave ...............63

(a) Ecuilibrium Radiation and Kirchoff's law..........64

(b) Planck's blackbody function ano related laws ........65

Excursus: Brightness temperature ............. 70

(c) Blackbodies and cavity radiometers ..............71

2.6 Notes and Comments ............................ 74

2.7 Problems $\ldots \ldots \ldots \ldots \ldots \ldots \ldots \ldots \ldots \ldots \ldots \ldots \ldots \ldots \ldots . . \ldots \ldots$

\section{Microscopic Interactions -}

Atomic and Molecular Absorption ........79

3.1 The atomic Absorption spectrum ..................79

(a) The Bright Line Spectrum......................79

(b) The Absorption Line Spectrum ..................82

3.2 Molecular Absorption Spectra ................... 83

(a) Molecular Bonding and the Molecular Dipole Moment .86

(b) Vibration and Rotation Spectra of simple

Diatomic Molecules: Illustrative Examples...........88

(c) Absorption by two triatomic gases.................93

Excursus: The Infra-red Carbon Dioxide Laser ........93 
3.3 Line Shapes ...................................97

(a) Pressure Brosdening ........................... 98

Excursus: Diatomic Molecules: Vertical

Distributions of Chlorine Monoxide................ 102

(b) Doppler Braadening........................... 105

3.4 Absorption Coefficients and Transmission Functions ...... 107

(a) The absorption Coefficient......................107

(b) Transmission Functions ...................... 108

3.5 The atmospheric Absorption spectrum ............. 113

(a) Molecular Oxygen ............................114

(b) Ozone.....................................116

(c) Carbon Dioxide............................. 117

(d) Water Vapor................................118

Excursus: The remote sensing of surface pressure ..... 119

3.6 Passive Spectrometer Systems ..................... 121

(a) Prism Spectrometers .......................... 121

(b) Grating Spectrometers........................ 124

(c) Interferometer Spectrometers....................128

Excursus: The resolving Power of an Interferometer...128

3.7 Notes and Comments ........................... 133

3.8 Problems . ................................. 137

\section{Macroscopic Interactions -}

Optical Properties ..........................141

4.1 Polarization of Matter $. . \ldots \ldots \ldots \ldots \ldots \ldots \ldots \ldots \ldots \ldots . \ldots \ldots 1$

4.2 Classical Theories ..............................144

(a) Lorentz Model ............................. 145

(b) Orientational Polarization- Debye Relaxation.........149

(c) Summary .............................. 153

4.3 The Refractive Index ..........................156

Excursus: The Refractive Index of Air and the 
Concentration of Atmospheric Oxygen .............159

4.4 Reflection and Transmission at a Plane Boundary ....... 161

(a) Propagation in a Homogeneous Slab............... 162

(b) Attenuation of Radiation in a Homogeneous Slab .... 166

4.5 Selected Applications of Surface Remote Sensing ........ 167

(a) Monitoring Sea Ice Extent....................... 168

(b) Measurement of near surface wind speed............170

Excursus: Reflection from Surfaces -

Bidirectional Reflection Functions ............... 175

Excursus: Spectral Reflectance Properties of

Land Surfaces ................................. 179

4.6 Notes and Comments $\ldots \ldots \ldots \ldots \ldots \ldots \ldots \ldots \ldots \ldots \ldots . \ldots \ldots 2$

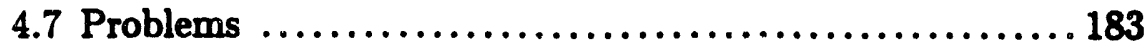

5. Macroscopic Interactions -

Particle Absorption and Scatte:ing ......189

5.1 A Single Oscillating Dipole: Rayleigh Scatter ............190

Excursus: Haidinger's Brush ..................196

5.2 Radiation from Multiple Dipoles ................... 197

(a) Scattering by Two Isolated Dipoles............... 197

Excursus: Phased Array Antennae ...............201

(b) The Discrete Dipole Approximation .............. 203

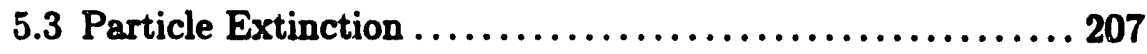

Excursus: Extinction - its black and white .........207

(a) Efficiency Factors and Cross-sectional Areas..........209

(b) Extinction by a cloud of many particles............210

(c) The Rayleigh Limit and its Application to cloud water retrieval.......................213

(d) The Large Particle Limit and the Extinction Daradox 
Excursus: Ship Tracks $\ldots \ldots \ldots \ldots \ldots \ldots \ldots \ldots \ldots \ldots 218$

5.4 Scattering Functions...........................220

(a) Amplitude Functions.......................221

(b) The Scattering Phase Function ................. 222

Excursus: A Polynomial Representation

of the Phase Function ...................... 225

(c) An Example of the Remote Sensing

of Ice C.ystal Phase functions....................227

5.5 A Simple Diffraction Theory

of Particle Extinction..............................229

Excursus: Particle Absorption in the

ADT Approximation ...................... 232

5.6 Scattering by Spheres:

A Brief Outline of Lorenz-Mie Theory ................ 234

5.7 Particle Backscattering ......................... 239

(a) Backscattering by Small Spheres..................240

(b) Backscatering by non-spherical particles...........241

5.8 Notes and Comments ........................ 247

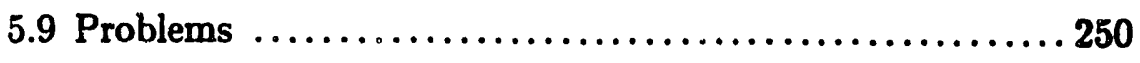

6. Passive Sensing -

Extinction and Scattering .................259

6.1 Beer's Law and the

Remote Sensing of Aerosol.........................259

(a) Aerosol Turbidity ........................... 261

(b) Measurement of Turbidity......................262

(c) Retrieval of Particle Size..................... 264

Excursus: Size Distributions from

Anomalous Diffraction Theory .................268

6.2 More on Extinction Based Methods ................. 270

(a) Total Ozone from UV Extinction Measurements ..... 270 
(b) Limb Profiling by Extinction-SAGE............273

6.3 Scattering as a Source of Radiation .................277

Excursus: Single Scatter Albedo

of Water and Ice Spheres .....................281

6.7 Multiple Scattering - a Natural Solution ..............285

Excursus: How Many Times does

a Photon get Scattered ..................... 287

Excursus: Primary Scattering in a

Simple Plane Parallel Atmosphere .............. 289

(a) An Example: An Atmospheric Correction for NDVI .. 290

(b) A Second Example: Aerosol from AVHRR..........291

6.5 The Remote Sensing of Ozone

from Scattered UV Radiation .......................... 294

(a) Reflection from below: The Principle of interaction ... 300

(b) Total Ozone Algorithms from Backscattered UV ...... 304

(c) Measured Trends in Total Ozone .................306

6.6 Sensing Clouds by Reflected Sunlight ............... 307

(a) Tne Two Stream Approximation ............... 306

Excursus: Pollution Susceptible Clouds ...........315

(b) Cloud Droplet Radii from Reflected Sunlight ........ 316

(c) Cloud Top Pressure from Reflected Sunlight ......... 317

6.7 Notes and Comments .......................... 231

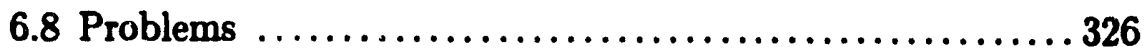

7. Passive Sensing - Emission ...............331

7.1 Radiative Transfer with Emission ................. 332

Excursus: The Angular Properties of

Emitted Radiation ................................... 325

7.2 The Remote Sensing of Sea Surface Temperature ........ 337

7.3 Examples of Path Integrated Quantities..............341 
(a) Microwave Radiative Transfer ....................343

Excursus: Surface-Level Humidity and

Heat Fluxes over the Ocean .................... 344

(b) A Microwave Method for Integrated Water

Vapor and Cloud Water ...................... 346

7.4 Passive Sensing of Rainfall

(a) Rainfall Estimates from Outgoing

(a) Longwave Radiation (OLR) ..................... 351

Microwave Emission Methods for Sensing Rainfall ....354

7.5 Principles of Sounding by Emission .................357

(a) Weighting Functions for Nadir Sounding ........... 359

(b) Weighting Functions for Zenith Viewing ........... 362

(c) Weighting Functions for Limb sounding ............354

(d) Weighting Functions of an Operational Sounder system 366

7.6 Sensing Clouds by Emission:

Windows and Arches ...........................370

Excursus: Golden Arches ........................376

7.7 More on Clouds:

Slicing up the Atmosphere .................... 377

7.8 Intensity Classification of Clouds ......................382

(a) Emission Classification in the Split Window .........383

(b) The International Satellite Cloud

Climatology Project (ISCCP) ...................362

7.9 Notes and Comments ......................... 389

7.10 Problems $\ldots \ldots \ldots \ldots \ldots \ldots \ldots \ldots \ldots \ldots \ldots \ldots \ldots \ldots \ldots . . . \ldots 91$

8. Active sensing . .........................397

8.1 Basic Operational considerations ................400

(a) Range Resolving Systems ..................400

(b) Scanning modes ........................402 402

8.2 Conventional Weather Radar .....................403 
(a) The Radar Equatiou .........................405

Excursus: Reflectivities and Precipitation .........410

(b) Radar Attenuation ....................... 413

(c) Area-Time Integral Methods for Convective Rain ... 417

8.3 Multi-Parameter Radar Measurements

of Rainfall.....................................422

(a) A Dual Wavelength Method ...................422

(b) Dual Polarization Method ..................... 424

8.4 General Principles of Lidar .......................29

(a) The Lidar Equation ........................ $\$ 22$

(b) Inversion of the Lidar Equation:

( Backscatter to Extinction Ratios ................432

(c) High Spectral Resolution Lidar ................435

(d) Multiple Scattering Effects .................. 438

8.5 The Differential Absorption Lidar ................438

8.6 Doppler Wind Measurements ....................442

Excursus: beating the Signal-

Heterodyne Detection .......................443

(a) General Principles of Doppler Wind Measurements ...445

(b) The Doppler Spectrum .....................446

8.7 Analyses of Doppler Measurements ................447

(a) Vertical Incidence ........................ 447

(b) Scanning Methods for Single Doppler Systems ...... 449

(c) Doppler Signatures of Horizontally Uniform Winds ...454

(d) Multiple Doppler Systems .................. 455

8.8 UHF/VHF Radars ............................457

(a) Echoing Mechanisms ...................... 457

(b) Wind Messurements ........................460

(c) Precipitation Echoes ........................462 
8.9 Notes and Comments $\ldots \ldots \ldots \ldots \ldots \ldots \ldots \ldots \ldots \ldots \ldots .462$

8.10 Problems .................................. 464

References ..................................469

Appendix 1: Frames of Reference ........495

1.1 Necessary Booking: Frames of Reference ............ 495

1.2 Scattering Angle $. . \ldots \ldots \ldots \ldots \ldots \ldots \ldots \ldots \ldots \ldots \ldots . \ldots 498$

1.3 Solid angle and Hemispheric Integrals ..............498

Appendix 2: Class Projects .............500

2.1 Interferometer Measurements of Atmospheric Emission ...500

(a) Procedures ..............................500

(b) Tasks $\ldots \ldots \ldots \ldots \ldots \ldots \ldots \ldots \ldots \ldots \ldots \ldots \ldots \ldots, \ldots \ldots \ldots$

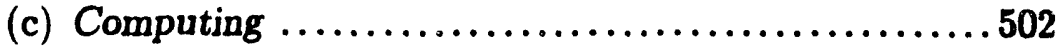

2.2 Turbidity at Mouna Loa $\ldots . \ldots \ldots \ldots \ldots \ldots \ldots \ldots \ldots . .503$

(a) Procedure and Tasks .......................503

(b) Computing ............................... 504

2.3 Retrieval of moisture Parameters using SSM/I data ......505

(a) Procedure and Tasks ......................505

(b) Data Information ...........................506

(c) Computing Comments ........................507

2.4 HIRS Temperature Sounding Project ...............508

(a) Weighting Functions .......................508

(b) Direct Inversion ............................ 509

(c) Interactive method of Inversion ................511

(d) Questions ...............................512

(e) Computing comments ..................... 513

(f) Additional References ..................... 513

2.5 Doppler velocity Project ........................... 508 
(a) Procedures $\ldots \ldots \ldots \ldots \ldots \ldots \ldots \ldots \ldots \ldots \ldots \ldots \ldots . \ldots, 508$

(b) Tasks $\ldots \ldots \ldots \ldots \ldots \ldots \ldots \ldots \ldots \ldots \ldots \ldots \ldots \ldots . \ldots \ldots$

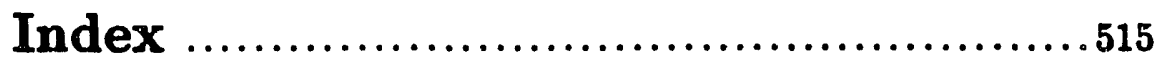



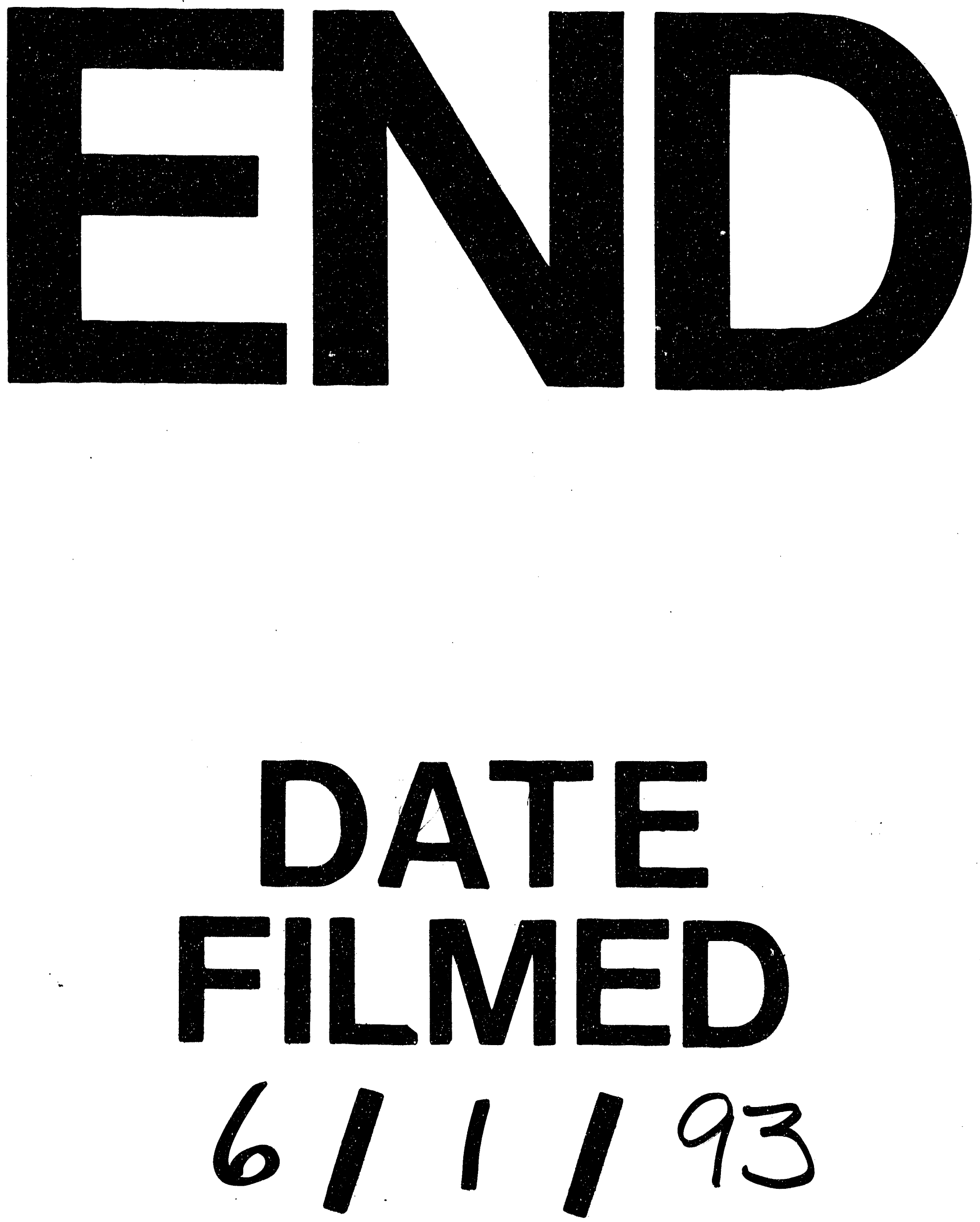
